\title{
BMJ Open Cohort profile: the Spanish Early-onset Colorectal Cancer (SECOC) cohort: a multicentre cohort study on the molecular basis of colorectal cancer among young individuals in Spain
}

Jose Perea (D) , ${ }^{1}$ Marc Marti, ${ }^{2}$ Eloy Espin, ${ }^{2}$ Sergio Hernandez-Villafranca, ${ }^{1}$ Pilar Orihuela, ${ }^{1}$ Rosario Vidal Tocino, ${ }^{3}$ Jose Antonio Alcazar, ${ }^{4}$ Alfredo Vivas, ${ }^{5}$ Cristina Narvaez, ${ }^{5}$ Isabel Prieto, ${ }^{6}$ Luis Asensio, ${ }^{6}$ Irene López Rojo, ${ }^{7}$ Sara Encinas Garcia, ${ }^{7}$ Elena Hurtado, ${ }^{8}$ Luis M Jiménez, ${ }^{8}$ Fernando Jiménez, ${ }^{9}$ Adriana Cavero, ${ }^{9}$ Edurne Alvaro, ${ }^{10}$ Maria Luisa Fuenmayor, ${ }^{10}$ Marta Jiménez Toscano, ${ }^{11}$ Mar Iglesias Comas, ${ }^{11}$ Francesc Balaguer, ${ }^{12,13}$ Maria Daca, ${ }^{13}$ Araceli Ballestero, ${ }^{14}$ Javier Die Trill, ${ }^{14}$ Gonzalo Sanz, ${ }^{15}$ Rodrigo Sanz López, ${ }^{15}$ Sirio Melone, ${ }^{16}$ Jose A Rueda, ${ }^{16}$ Lorena Brandariz, ${ }^{17}$ Ignacio Valverde, ${ }^{17}$ Jorge Arredondo, ${ }^{18}$ Carlos Pastor, ${ }^{19}$ Damian Garcia-Olmo, ${ }^{1,20}$ Nuria Malats, ${ }^{21}$ Miguel Urioste, ${ }^{21}$ Rogelio Gonzalez-Sarmiento, ${ }^{22}$ Antonino Spinelli, ${ }^{23,24}$ Andreana N Holowatyj, ${ }^{25,26}$ on behalf of the Spanish EOCRC group and The Spanish Early-onset Colorectal Cancer (SECOC) Consortium

To cite: Perea J, Marti M, Espin E, et al. Cohort profile: the Spanish Early-onset Colorectal Cancer (SECOC) cohort: a multicentre cohort study on the molecular basis of colorectal cancer among young individuals in Spain. BMJ Open 2021;11:e055409. doi:10.1136/ bmjopen-2021-055409

- Prepublication history for this paper is available online. To view these files, please visit the journal online (http://dx.doi. org/10.1136/bmjopen-2021055409).

Received 11 July 2021

Accepted 16 December 2021

Check for updates

(c) Author(s) (or their employer(s)) 2021. Re-use permitted under CC BY-NC. No commercial re-use. See rights and permissions. Published by BMJ.

For numbered affiliations see end of article.

Correspondence to Dr Jose Perea; josepereag@hotmail.com

\section{ABSTRACT}

Purpose The Spanish Early-onset Colorectal Cancer (SECOC) study is a multicentre prospective cohort established in Spain to investigate the molecular basis of early-onset colorectal cancer (EOCRC), including metabolic alterations.

Participants 220 patients with EOCRC have been enrolled since January 2019 through 18 centres across Spain. Individual-level data were collected by questionnaire, including lifestyle and other colorectal cancer-related factors. Medical record review was performed to capture clinical, histopathological and familial cancer history data. Biospecimen collection (blood, stool, tissue) at diagnosis and at various time points across treatment, as applicable, is also completed.

Findings to date Participants had a median age of 44 years (range 14-49), and the majority are men $(60 \%)$, with individuals age $40-49$ years at EOCRC diagnosis being over-represented. Forty-three per cent of participants were diagnosed with a tumour in the rectosigmoid junction/rectum. Nearly two-thirds of EOCRC cases (64\%) were diagnosed with advanced stage (III-IV) disease, and $28 \%$ of cases had no reported familial history of cancer.

Future plans We are actively recruiting and observing participants; we plan to administer follow-up questionnaires and perform additional biospecimen collection. This prospective cohort offers a unique, rich resource for research on EOCRC aetiologies and will contribute to larger international efforts to disentangle the rising disease burden.

\section{Strengths and limitations of this study}

- One of the main strengths of Spanish Early-onset Colorectal Cancer study is the prospective cohort design, which reduces different type of bias.

- The demographic composition of this cohort is predominantly European white, being at the same time a weakness, leaving aside ethnic minorities to take into account in Spain (Latino, etc), but it can also be a strength, by minimising any potential difference in the findings due to the marked disparities of the early-onset colorectal cancer (EOCRC).

- The cohort covers a wide range of aspects of the EOCRC (clinical, family, histological features), important when analysing their relationships with the molecular analyses to be developed.

- The collaboration with patient advocacy organisations in Spain and Europe confers an essential perspective on the priorities for patients.

\section{INTRODUCTION}

Colorectal cancer (CRC) is the third most commonly diagnosed cancer worldwide $(10 \%$ of all new cases, excluding non-melanoma skin cancer), and ranks as the second leading cause of cancer-related deaths. ${ }^{1}$ Despite a reduction in the absolute numbers of patients diagnosed with CRC, mainly due to advances and uptake in screening modalities-particularly in developed countries-there has 
been a remarkable increase in CRC incidence among individuals younger than age 50 years at diagnosis in many countries ${ }^{2}$ (early-onset CRC; EOCRC). In fact, by 2030 , incidence rates for colon and rectal tumours in the USA are estimated to increase by $90.0 \%$ and $124.2 \%$, respectively, for patients aged 20-34 years and by $27.7 \%$ and $46.0 \%$, respectively, for patients aged $35-49$ years. ${ }^{34}$ Moreover, CRC incidences and deaths in the younger age group (20-49 years) will continue on in the next two decades, becoming the second leading cancer in this age group. By 2040, the top four estimated cancers in this age group for both male and female individuals combined will be breast, colorectal, thyroid and kidney and renal pelvis, and the top four cancer-related deaths in this age group are estimated again colorectal and breast, together with lung and brain or other central nervous system. ${ }^{5}$ As such, EOCRC stands as a global public health problem that warrants intervention in order to reverse these alarming trends.

In Europe, heterogenous patterns of EOCRC incidence have been reported, with increasing incidence regarding EOCRC in countries with a stable or declining trend among adults older than 50 (including Germany, the UK, Denmark, Slovenia and Sweden); countries with an increasing CRC incidence among adults older than age 50 years (including Cyprus, the Netherlands and Norway), but with a twofold higher incidence of CRC in younger adults comparatively; however, across Italy, Austria and Lithuania, we are seeing a declining incidence in EOCRC. ${ }^{6}$ Interestingly, in Italy an increase in EOCRC incidence in a metropolitan area like Milan have been also seen, ${ }^{8}$ raising the possible existence of intracountry discrepancies, a situation that, due to similarity, could also be seen in Spain. This unique epidemiology of EOCRC in Europe and also worldwide has led to increasing efforts to unravel aetiologies underlying these trends, and an imminent need to tailor clinical approaches for younger patients-including updated guidelines for decreasing routine screening ages, particularly in regions with a rising EOCRC burden.

Evidence is accumulating to suggest that EOCRC harbours a distinct clinical and molecular phenotype compared with CRCs diagnosed among individuals aged 50 years and older. ${ }^{9-11}$ While the proportion of hereditary CRC among young individuals seems to remain stable, the rise of sporadic EOCRC with aetiologies unexplained is alarming. To date, it is well established that increased EOCRC risk among individuals includes a marked family history of $\mathrm{CRC},{ }^{12}$ as well as a possible linkage to excess weight/adiposity and carbohydrate metabolism disorders. ${ }^{13}$ However, beyond these features, studies are emerging to suggest that lifestyle-related factors and early life exposures, also known as the exposome can influence the gut microbiome, leading to dysbiosis, a direct effect on mechanisms that lead to CRC development, by means of, between others, inflammation and fat tissue metabolism. ${ }^{14}$ Nevertheless, these factors are complicated by disproportionate burden of EOCRC across diverse population subgroups (eg, sex, race/ethnicity) that shed light on the multifactorial interplay of biology, genetics, behaviour and social determinants of health in EOCRC that must be considered in order to elucidate aetiologies underlying this disease. ${ }^{15}$

To address these timely and critical gaps of knowledge related to the rising EOCRC burden, we established a prospective cohort study that recruit individuals diagnosed with EOCRC across multiple regions of Spain. This cohort will serve as a robust resource, including biological samples collected uniformly at multiple time points, together with individual-level lifestyle and other CRC-related factors, clinicopathological, familial cancer history and follow-up data, for EOCRC. Furthermore, this will also help to raise awareness of the need for a better knowledge of the epidemiological situation of the EOCRC in Spain. Together, this well-characterised cohort will afford new opportunities and avenues to explore the molecular basis of EOCRC in order to develop a personalised management, diagnostic and more important, preventive strategies to reduce this disease burden.

\section{COHORT DESCRIPTION}

\section{Study design and population}

The Spanish Early-onset Colorectal Cancer (SECOC) cohort was established to collect high-quality data on individual-level characteristics using a questionnaire paired with biological fresh samples for molecular study. SECOC started recruitment in January 2019, and to date has enrolled 220 patients with a pathologically confirmed CRC diagnosis before age 50 years. Inclusion criteria are patients diagnosed with CRC younger than age 50 years, no history of inflammatory bowel disease and a histopathological diagnosis of adenocarcinoma. Affiliated centres $(n=18)$ enrolling patients into SECOC are depicted in figure 1 .

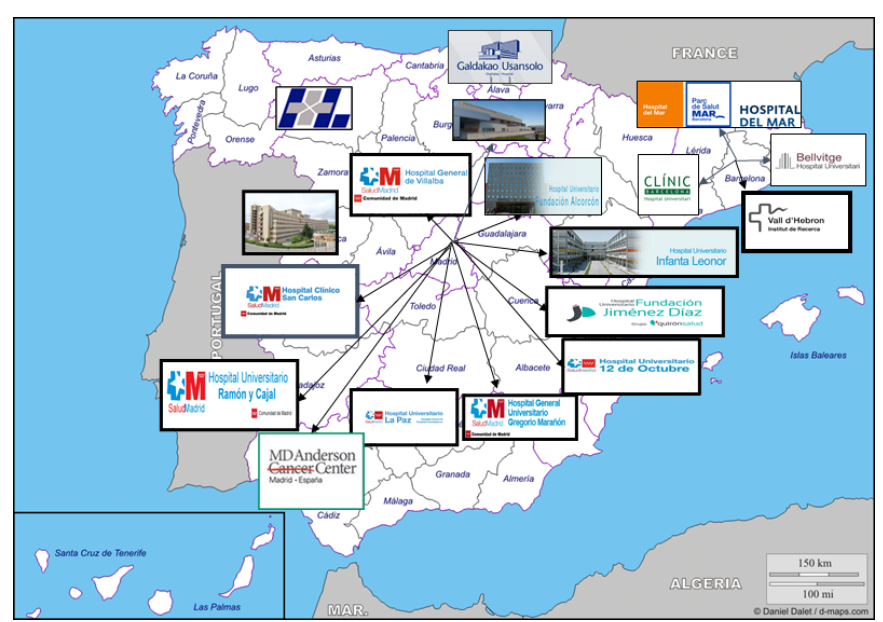

Figure 1 Distribution of Spanish Early-onset Colorectal Cancer Consortium participating centres across Spain. 
Box 1 Overview of questionnaire data collected for the Spanish Early-onset Colorectal Cancer cohort

\section{Questionnaire \\ Demographic information \\ - Race/ethnicity. \\ - Country of birth (duration of residence in Spain). \\ Anthropometrics \\ - Body mass index (at diagnosis, at age 18 years). \\ - Area of the body where fat accumulates with weight gain. \\ Lifestyle characteristics during childhood and adulthood \\ - Smoking. \\ - Alcohol use. \\ - Physical activity. \\ - Medication history (anti-inflammatory drugs, steroids, antibiotics, probiotics).}

\section{Dental history}

\section{Dietary intake in the last 2 years}

- Food frequency.

- Significant changes to diet in previous decade.

\section{Medical history}

- Pathologies or diseases (type and duration).

- Medications or environmental allergies.

- Previous surgeries.

\section{Familial history}

- Diseases not related to cancer (type, relationship, age, death).

- Cancer (type, relationship, age, death).

\section{Reproductive health (women)}

- Menarche.

- Menopausal status.

- Pregnancy, childbirth history and conditions.

- Children.

- Oral contraceptive use (age, duration, type).

\section{Data collection}

\section{Questionnaire data}

An outline of the topics included in the SECOC baseline questionnaire, administered prior to CRC surgery, are listed in box 1. Briefly, these include demographic characteristics, medical history, anthropometrics including body mass index (BMI) at diagnosis and age 18 years, lifestyle-related factors (eg, smoking, alcohol, drug/ medication use, dental history, physical activity), dietary intake, familial cancer and medical history, as well as reproductive health for women. For familial cancer history, pedigrees were collected with at least two additional generations from the proband and classified into first and second degree relatives. For each category, cases were classified according to the presence of: CRC family history; digestive cancers other than CRC; gynaecologic and/or breast cancers; urinary tract and prostate cancers; and other tumour type. Cases were defined as having a second degree relative when only this fact was present and none in first degree relative presented cancer. In addition, on identification of a family cancer history, data were captured to ascertain presence/absence of earlyonset cancers $(<50$ years of age). And finally, cases were classified as sporadic if they did not present with any first or second degree relatives with a history of cancer.

\section{Clinicopathological data}

Clinicopathological and pathological characteristics were ascertained from detailed medical record review. Variables include age at CRC diagnosis, sex, anatomical tumour location, tumour grade, mucin production, signet ring cells, perineural and vascular invasion, tumour budding, tumour infiltrating lymphocytes, tumour stage lymph node involvement, metastasis site(s), CRC treatments, BMI at diagnosis, medical and detailed information on colorectal polyps detected before/at EOCRC diagnosis. Presence of multiple primary neoplasms (eg, synchronous and metachronous CRC) was also captured and defined as follow: (i) Synchronous CRC was defined as two or more histologically different CRCs that developed simultaneously or within 6 months of detection of the first CRC diagnosis; and (ii) Metachronous CRC was defined on presence of a secondary neoplasm detected distant from the anastomosis area, greater than 6 months after first CRC diagnosis.

\section{Follow-up data ascertainment}

Follow-up is completed for all EOCRC cases in SECOC every 6 months. In addition to surveillance for disease outcomes, cases are followed for the presence of colorectal polyps after EOCRC diagnosis. ${ }^{16}$

\section{Biological sample collection}

Given the overarching goal of SECOC to characterise the molecular basis of EOCRC, biological fresh samples are collected at multiple time points in this study. Timing and types of sample collection for SECOC are depicted in figure 2. Notable, early-onset rectal cancer cases who receive neoadjuvant therapy have a distinct biological sample collection pattern in order to collect samples prior to tumour therapy.

The collection of faecal (stool) samples from patients with EOCRC affords a unique opportunity to study the

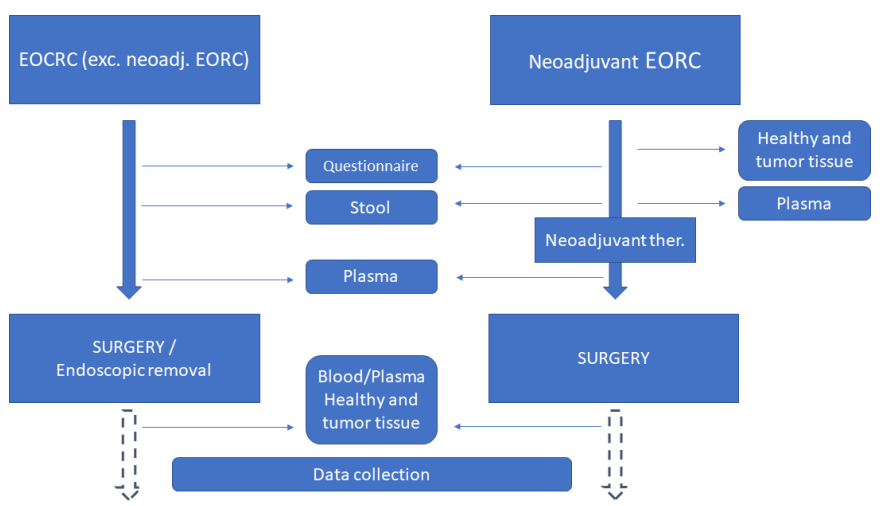

Figure 2 Study data and biospecimen collection diagram. EOCRC, early-onset colorectal cancer; EORC, early-onset rectal cancer. 
microbial diversity within this patient population by $16 \mathrm{~S}$ and/or metagenomic sequencing. The collection of stool is assembled as far out as possible from the diagnostic colonoscopy, and prior to admission for CRC treatment, to avoid possible contamination from bowel preparations. Blood collection includes whole blood as well as plasma samples for future molecular-omics studies. Routine tissue collection includes both normal (healthy) colon and tumour tissue collected during endoscopy or surgery (the main proportion of samples).

As mentioned before, different protocol will be assessed for rectal cancer cases receiving neoadjuvant therapy. In this case, an additional sample collection will be defined prior to that therapy, collecting both tumour and healthy colon tissues and plasma sample. In these cases, stool collection will be made also before neoadjuvant therapy.

Finally, as we progress in the recruitment and based on the preliminary results, the consequent sample calculations will be carried out, and thus the different types of samples and necessary data will be able to be carried out.

\section{Definition and collection of control group}

The control group is being selected in a ratio of 2:1 in relation to cases. They are healthy subjects under 50 years of age and matched with the cases in defined characteristics. The match process is defined by the following case variables: age at the EOCRC diagnosis, sex, race and geographical area of diagnosis. In addition, all colonic pathology (inflammatory bowel disease, etc) will be exclusion criteria for cases, as well as any diagnosis of cancer in the controls, or suspicion of hereditary syndrome in the family. For controls, initially stool samples is being also collected, as well as they fulfil the lifestyles questionnaire.

\section{Patient and public involvement}

No patient involved until now, but the immediate steps will be to include in the coordinating committee of the cohort both representation of patients, as well as patient organisations, not only from Spain, but also European, in order to make the results known constantly, and expand the knowledge accordingly, but also to receive feedback on the needs of those to adapt future studies in this regard. For this reason, Digestive Cancers Europe (www.digestivecancers.eu), the European umbrella organisation of a large group of national members representing patients with digestive cancer-colorectal, gastric, pancreatic and rare cancers, has been already contacted. In addition, there will be a need to expand the results not only scientifically but publicly to raise awareness of this problem in society, as well as the importance of future educational strategies both in the medical community and in society in general.

\section{FINDINGS TO DATE}

Baseline demographic characteristics

Individual and clinicopathological characteristics for a subset ( $n=176$ of 220) of currently enrolled EOCRC cases with annotated data in SECOC are provided in table 1. Among these cases, the majority $(60.3 \%)$ were menwhich is aligned with a higher incidence of CRC among men. At baseline, patients with EOCRC in SECOC have a median age of 44 years, with a large proportion of the cohort diagnosed between ages 40 and 49 years (74\%). Four out of every 10 patients with EOCRC in SECOC $(42.6 \%)$ had a BMI classified as overweight or obese. Less than one-third of cases $(28.4 \%)$ had no family history of cancer, while another one-third (31.3\%) of cases had positive family CRC history.

\section{EOCRC clinicopathology}

The most frequent tumour location among cases was the rectum $(42.6 \%)$, followed by tumours in the left colon and then right colon (32.4\% and $25 \%$, respectively). Nearly two-thirds of cases $(64.2 \%)$ were diagnosed with advanced stage disease (stage III-IV). Twenty-one per cent of colorectal adenocarcinomas among patients with EOCRC harboured a mucinous component.

\section{Molecular characterisation of EOCRC}

Given the emerging role of metabolic dysregulation in EOCRC, ${ }^{7}$ the first molecular characterisation of case in SECOC is to assess metabolic alterations, including carbohydrate metabolism, in EOCRC via transcriptomic (RNA-sequencing) profiling paired with detailed lifestylerelated data. Profiling of tumorous tissues is currently underway and anticipated to be paired with additional omics profiles in the future to provide a multidimensional characterisation of the molecular basis of EOCRC.

\section{STRENGTHS AND LIMITATIONS}

We established the SECOC cohort, the largest prospective study on EOCRC with a nationwide distribution in Spain. The detail and breadth of data on various exposures and biological samples collected is presented in this paper. This uniform collection enables unique and in-depth molecular characterisation of EOCRC, and more complex multiomics analyses, in order to elucidate underlying aetiologies of this rising disease burden. In addition, our ongoing partnership with patient advocacy organisations in Spain and Europe provides a unique perspective and insight on the priorities and needs for patients in order to tailor SECOC and expand the impact of this study moving forward.

Another strength of SECOC is the prospective cohort design, which reduces selection and recall bias among EOCRC cases. Moreover, identical protocols/data and biospecimen collection across all 18 centres in SECOC allows for uniform data pooling and harmonisation, as well as the ability to assess geographical heterogeneity of EOCRC across Spain. ${ }^{13}$ This is particular advantage for biospecimen collection, as it will allow for the characterisation and integration of molecular assays and analyses to deepen our understanding of the molecular basis of EOCRC. However, future expansion of biological sample 
Table 1 Baseline characteristics of the patients with earlyonset CRC cohort: Spanish Early-onset Colorectal Cancer (SECOC) Consortium

\begin{tabular}{|c|c|}
\hline \multirow[b]{2}{*}{ Characteristic } & \multirow{2}{*}{$\begin{array}{l}\text { Total } \\
\text { N (\%) }\end{array}$} \\
\hline & \\
\hline Total & 176 \\
\hline \multicolumn{2}{|l|}{ Sex } \\
\hline Female & $70(39.7)$ \\
\hline Male & $106(60.3)$ \\
\hline \multicolumn{2}{|l|}{ Age at diagnosis } \\
\hline$<30$ years & $6(3.4)$ \\
\hline 30-39 years & 39 (22.2) \\
\hline 40-49 years & $131(74.4)$ \\
\hline Mean (std), years & $42.7(6.2)$ \\
\hline Median (range), years & $44(14-49)$ \\
\hline \multicolumn{2}{|l|}{ Body mass index } \\
\hline Underweight $\left(<18.5 \mathrm{~kg} / \mathrm{m}^{2}\right)$ & $9(5.1)$ \\
\hline Normoweight $(18.5-<25$ kg/m²) & $62(35.2)$ \\
\hline Overweight $(25-<30$ kg/m²) & $56(31.8)$ \\
\hline Obese $\left(30+\mathrm{kg} / \mathrm{m}^{2}\right)$ & $19(10.8)$ \\
\hline Unknown & $30(17.1)$ \\
\hline Mean (std), kg/m² & 25.6 \\
\hline Median (range), kg/m² & $25.2(14.2-43)$ \\
\hline \multicolumn{2}{|l|}{ Tumour stage at diagnosis } \\
\hline 1 & $26(14.8)$ \\
\hline II & $29(16.5)$ \\
\hline III & $75(42.6)$ \\
\hline IV & $38(21.6)$ \\
\hline Unknown & $8(4.5)$ \\
\hline \multicolumn{2}{|l|}{ Tumour site } \\
\hline Right colon & $44(25)$ \\
\hline Left colon & $57(32.4)$ \\
\hline Rectosigmoid junction/rectum & $75(42.6)$ \\
\hline \multicolumn{2}{|l|}{ Histological features ${ }^{*}$} \\
\hline Mucinous & $34 / 162(21)$ \\
\hline 'Signet ring' cells & $7 / 162(4.3)$ \\
\hline Unknown & $14(8)$ \\
\hline \multicolumn{2}{|l|}{ Grade of differentiation* } \\
\hline Low & 18 (10.2) \\
\hline Medium & $84(47.7)$ \\
\hline High & $62(35.2)$ \\
\hline Unknown & $12(6.8)$ \\
\hline \multicolumn{2}{|l|}{ History of polyps } \\
\hline None & $113(64.2)$ \\
\hline Yes & 57 (32.4) \\
\hline Adenomatous & 33 (57.9) \\
\hline Hyperplastic & $8(14)$ \\
\hline Serrated & $3(5.3)$ \\
\hline Mixed & $11(19.3)$ \\
\hline Unknown & $2(3.5)$ \\
\hline Unknown & $6(3.4)$ \\
\hline
\end{tabular}

Continued

\section{Table 1 Continued}

\begin{tabular}{ll}
\hline & Total \\
\cline { 2 - 2 } Characteristic & N (\%) \\
\hline Multiple primary neoplasms & \\
Synchronous CRC & $6(3.4)$ \\
Other & $13(7.4)$ \\
\hline Familial CRC history & \\
Yes & $55(31.3)$ \\
\hline No & $113(64.2)$ \\
\hline Unknown & $8(4.5)$ \\
\hline Familial cancer history & \\
\hline Yes & $50(28.4)$ \\
\hline No & $122(69.3)$ \\
\hline Unknown & $4(2.3)$ \\
\hline Microsatellite instability & \\
\hline MSS & $112(63.6)$ \\
\hline MSI & $16(9.1)$ \\
\hline Unknown & $48(27.3)$ \\
\hline
\end{tabular}

${ }^{*}$ Cases with only a biopsy due to palliative care are excluded from tumour histology/grade.

CRC, colorectal cancer; MSI, microsatellite instability; MSS, microsatellite stability.

collection-including among cases receiving adjuvant therapy, will expand the potential of SECOC to define changes in molecular features caused by these therapies and may yield insight into potential biomarkers for disease recurrence and prognostic outcomes. This will also afford a unique opportunity to continue building a robust cohort size with sufficient statistical power that will allow for clinical translation of our findings in the future (eg, liquid biopsy).

While SECOC is the first-of-its-kind cohort across Spain, it should be noted that the demographic composition of this cohort is predominantly comprised of European whites. While this will minimise any potential differences in biological findings due to the marked EOCRC racial/ethnic disparities, ${ }^{15} 17$ collaborations with other studies worldwide that include an over-representation of diverse population subgroups will be essential in order to better understand the disproportionate burden of EOCRC moving forward. In addition, the inclusion of SECOC in larger, international consortium efforts will also overcome this emerging limitation and allow for and increased sample size of EOCRC cases.

\section{COLLABORATIONS}

The SECOC multicentre prospective cohort will continue to collect a robust set of data, including follow-up, and biological samples. Researchers interested in collaboration are invited to propose EOCRC research based on the data/biospecimen available within SECOC or submit a request for additional data collection. Requests can be submitted to JP (josepereag@hotmail.com) and will be 
reviewed by the SECOC committee. Moreover, SECOC is a contributing cohort to the Global Early-Onset Colorectal Cancer (GEOCODE) Consortium-in order to define the burden and patterns of EOCRC worldwide, including EOCRC disparities, and is open to further collaborations with other EOCRC cohorts toward this objective. Other ongoing EOCRC projects, like BEYOND project (BEtter understanding of Young-ONset ColoRectal Cancer) ${ }^{18}$ with a similar range of sample collection, could be good candidates to explore synergies in the discovery of the mechanistic of the EOCRC and its molecular bases.

\section{Author affiliations}

${ }^{1}$ Surgery Department, Fundacion Jimenez Diaz-UTE, Madrid, Spain

${ }^{2}$ Surgery Department, Vall d'Hebron University Hospital, Barcelona, Catalunya, Spain ${ }^{3}$ Oncology Department, University Hospital of Salamanca, Salamanca, Castilla y León, Spain

${ }^{4}$ Surgery Department, University Hospital of Salamanca, Salamanca, Castilla y León, Spain

${ }^{5}$ Surgery Department, Hospital Universitario 12 de Octubre, Madrid, Comunidad de Madrid, Spain

${ }^{6}$ Surgery Department, La Paz University Hospital, Madrid, Spain

${ }^{7}$ Surgery Department, MD Anderson Cancer Center Madrid, Madrid, Comunidad de Madrid, Spain

${ }^{8}$ Surgery Department, General University Hospital Gregorio Maranon, Madrid, Spain

${ }^{9}$ Surgery Department, Galdacano Hospital, Galdacano, Spain

${ }^{10}$ Surgery Department, Hospital Universitario Infanta Leonor, Madrid, Spain

${ }^{11}$ Surgery Department, Hospital del Mar, Barcelona, Catalunya, Spain

${ }^{12}$ Institut d'Investigacions Biomèdiques August Pi i Sunyer (IDIBAPS), Barcelona, Spain

${ }^{13}$ Gastroenterology Department, Hospital Clinic de Barcelona, Barcelona, Catalunya, Spain

${ }^{14}$ Surgery Department, Ramon y Cajal University Hospital, Madrid, Spain

${ }^{15}$ Surgery Department, San Carlos University Hospital, Madrid, Spain

${ }^{16}$ Surgery Department, Hospital Universitario Fundacion Alcorcon, Alcorcon, Madrid, Spain

${ }^{17}$ Surgery Department, Villalba General Hospital, Collado Villalba, Comunidad de Madrid, Spain

${ }^{18}$ Surgery Department, University Hospital Centre León, Leon, Spain

${ }^{19}$ Surgery Department, University of Navarra Clinic, Madrid, Spain

${ }^{20}$ Universidad Autonoma de Madrid, Madrid, Spain

${ }^{21}$ Spanish National Cancer Research Centre, Madrid, Spain

${ }^{22}$ Medicine Department, University of Salamanca, Salamanca, Spain

${ }^{23}$ Humanitas University, Rozzano, Lombardia, Italy

${ }^{24}$ IRCCS Humanitas Research Hospital, Rozzano, Lombardia, Italy

${ }^{25}$ Vanderbilt University Medical Center, Nashville, Tennessee, USA

${ }^{26}$ Vanderbilt-Ingram Cancer Center, Nashville, Tennessee, USA

Twitter Fernando Jiménez @fernandjimenez, Adriana Cavero @aci23_88 and Andreana N Holowatyj @drholowatyj

Collaborators Spanish EOCRC cohort (SECOC). Members and Affiliations: Belvitge University Hospital; Bellvitge Research Institute (IDIBELL), Barcelona: Principal Investigator: Cristina Santos. Associated Investigators: Mercedes Martínez Villacampa, Víctor Moreno, Jose Carlos Ruffinelli. Complutense University, School of Medicine, Madrid, Biostatistics Department: Principal Investigator: Lucía Inglada-Pérez. Fundación Alcorcón Hospital, Madrid: Principal Investigator: Sirio Melone. Associated Investigator: José A. Rueda Orgaz. Fundación Jiménez Díaz University Hospital, Madrid: Principal Investigator: José Perea. Associated Investigators: Víctor Castellano Megías, Damián García-OImo, Sergio HernándezVillafranca, Susana Olmedillas-López, Pilar Orihuela. Galdakao-Usansolo Hospital, Vizcaya: Principal Investigator: Fernando Jiménez. Associated Investigators: Adriana Cavero, Vicente Portugal. Gregorio Marañon University Hospital, Madrid: Principal Investigator: Elena Hurtado Caballero. Associated Investigators: María Arriba Domenech, Luis M. Jiménez Gómez, Isabel Peligros Gómez, Cristina Rey Valcárcel, Jaime Zorrilla Ortúzar. Department of Gastroenterology, Hospital Clínic de Barcelona, Institut d'Investigacions Biomèdiques August Pi i Sunyer (IDIBAPS), Centro de Investigación Biomédica en Red de Enfermedades Hepáticas y Digestivas
(CIBEREHD), University of Barcelona, Barcelona, Spain: Principal Investigator: Francesc Balaguer. Associated Investigators: María Daca, Teresa Ocaña, Miriam Cuatrecasas, Sabela Carballal, Leticia Moreira, Lorena Moreno, María Pellisé, Ariadna Sánchez. Hospital del Mar, Barcelona: Principal Investigator: Marta Jiménez Toscano. Associated Investigators: Mar Iglesias Coma. Molecular Oncology IMDEA Food Institute, Madrid: Principal Investigator: Ana Ramírez de Molina. Associated Investigators: Gonzalo Colmenarejo, Isabel Espinosa-Salinas, Lara Fernández, Marta Gómez de Cedrón. Biomedical Research Institute of Salamanca (IBSAL): Principal Investigator: Rogelio González-Sarmiento. Associated Investigators: Luis Corchete; Juan L. García; Paula García Vallés; Ana B. Hernández; Abel J. Martel Martel, Jéssica Pérez. Infanta Leonor University Hospital, Madrid: Principal Investigator: Edurne Álvaro. Associated Investigators: Ana Burdaspal, Maria Luisa de Fuenmayor. La Paz University Hospital, Madrid: Principal Investigator: Isabel Prieto (Surgery Department). Associated Investigators: Alejandro Forero, Inés Rubio. León University Hospital, León: Principal investigator: Jorge Arredondo (Surgery Department). Associated Investigators: Enrique Pastor, Jesús Fernández, Amaya Villafañe. MD Anderson Spain, Madrid: Principal Investigator: Irene López Rojo. Associated Investigators: Oscar Alonso, Sara Encinas, Ana Teijo. Navarra University Clinic, Madrid: Principal Investigator: Carlos Pastor. Associated Investigators: Jorge Baixauli Fons, Lucia Ceniceros Paredes, Javier Rodriguez Rodriguez Carlos Sánchez Justicia. Ramón y Cajal University Hospital: Principal Investigator: Araceli Ballestero Pérez. Associated Investigators: Javier Die Trill. San Carlos University Hospital, Madrid: Principal Investigator: Gonzalo Sanz (Surgery Department). Associated Investigators: Jana Dziakova, Sara Picazo Marín, Rodrigo Sanz López, María Suárez Solís. Salamanca University Hospital: Principal Investigator: José A. Alcazar. Associated Investigators: Jacinto García, Rosario Vidal Tocino. Spanish National Cancer Research Center. Clinical Cancer Unit. Human Genetics Group: Principal Investigator: Miguel Urioste. Spanish National Cancer Research Center. Genetic \& Molecular Epidemiology Group: Principal Investigator: Núria Malats. Associated Investigators: Lidia Estudillo. Secugen, Madrid: Principal Investigator: Julián PérezPérez. Vall d' Hebron University Hospital, Barcelona: Principal Investigator: Marc Martí Gallostra. Associated Investigators: Eloy Espín, Franco Marinello, Miquel Kraft, Stefania Landolfi. Villalba University Hospital, Madrid: Principal Investigator: Lorena Brandáriz. Associated Investigators: Ignacio Valverde. 12 de Octubre University Hospital, Madrid: Principal Investigator: Alfredo Vivas. Associated Investigators: Cristina Narváez, Inmaculada Salces, Sandra Tapial.

Contributors All authors are principal or associate investigators of SECOC project. MM, EE, SH-V, PO, RVT, JAA, AV, CN, IP, LA, ILR, SEG, EH, LMJ, FJ, AC, EA, MLF, MJT, MIC, FB, MD, AB, JDT, GS, RSL, SM, JAR, LB, IV, JA, CP, DG-0, NM, MU and RG-S are involved in the acquisition of data. JP designed and coordinates the study. JP, AS and ANH supervised the concept and design of the manuscript. JP is guarantor. All authors critically revised the manuscript and contributed to interpretation of the data. All authors read and approved the final version of the manuscript.

Funding This work was funded by Projects PI16/01650 and PI20/00974 to JP, from the Spanish Ministry of Health and Consumer Affairs and FEDER. ANH was supported by the National Institutes of Health (NIH) K12 HD043483 from the Eunice Kennedy Shriver National Institute of Child Health and Human Development. This work was also supported by the American Cancer Society (\#IRG-19-139-59) to ANH.

Map disclaimer The inclusion of any map (including the depiction of any boundaries therein), or of any geographical or locational reference, does not imply the expression of any opinion whatsoever on the part of BMJ concerning the legal status of any country, territory, jurisdiction or area or of its authorities. Any such expression remains solely that of the relevant source and is not endorsed by BMJ. Maps are provided without any warranty of any kind, either express or implied.

Competing interests None declared.

Patient and public involvement Patients and/or the public were involved in the design, or conduct, or reporting, or dissemination plans of this research. Refer to the Cohort description section for further details.

Patient consent for publication Not applicable.

Ethics approval This study was approved by the Fundación Jiménez Díaz University Hospital Ethics Board (PI20/00974), and the contributing centres to SECOC were approved by all local research ethics review committees, and participants signed and informed consent for SECOC prior to enrolment. Participants gave informed consent to participate in the study before taking part.

Provenance and peer review Not commissioned; externally peer reviewed.

Data availability statement Data are available upon reasonable request. Data will be available on reasonable request. Researchers may request access data 
by applying to the SECOC access committee. Nowadays, the best approach is connecting with the author for correspondence. Data also will be distributed in the corresponding repositories, according to each type of sample and analysis.

Open access This is an open access article distributed in accordance with the Creative Commons Attribution Non Commercial (CC BY-NC 4.0) license, which permits others to distribute, remix, adapt, build upon this work non-commercially, and license their derivative works on different terms, provided the original work is properly cited, appropriate credit is given, any changes made indicated, and the use is non-commercial. See: http://creativecommons.org/licenses/by-nc/4.0/.

\section{ORCID iD}

Jose Perea http://orcid.org/0000-0001-5522-8844

\section{REFERENCES}

1 Sung H, Ferlay J, Siegel RL, et al. Global cancer statistics 2020: GLOBOCAN estimates of incidence and mortality worldwide for 36 cancers in 185 countries. CA Cancer J Clin 2021;71:209-49.

2 Siegel RL, Fedewa SA, Anderson WF, et al. Colorectal cancer incidence patterns in the United States, 1974-2013. J Natl Cancer Inst 2017;109:djw322.

3 Ashktorab H, Kupfer SS, Brim H, et al. Racial disparity in gastrointestinal cancer risk. Gastroenterology 2017;153:910-23.

4 Bailey CE, Hu C-Y, You YN, et al. Increasing disparities in the agerelated incidences of colon and rectal cancers in the United States, 1975-2010. JAMA Surg 2015;150:17-22.

5 Rahib L, Wehner MR, Matrisian LM, et al. Estimated projection of US cancer incidence and death to 2040. JAMA Netw Open 2021;4:e214708.

6 Siegel RL, Torre LA, Soerjomataram I, et al. Global patterns and trends in colorectal cancer incidence in young adults. Gut 2019;68:2179-85.
7 Vuik FE, Nieuwenburg SA, Bardou M, et al. Increasing incidence of colorectal cancer in young adults in Europe over the last 25 years. Gut 2019;68:1820-6.

8 Russo AG, Andreano A, Sartore-Bianchi A, et al. Increased incidence of colon cancer among individuals younger than 50 years: a 17 years analysis from the cancer registry of the municipality of Milan, Italy. Cancer Epidemiol 2019;60:134-40.

9 Holowatyj AN, Gigic B, Herpel E, et al. Distinct molecular phenotype of sporadic colorectal cancers among young patients based on Multiomics analysis. Gastroenterology 2020;158:1155-8.

10 Archambault AN, Su Y-R, Jeon J, et al. Cumulative burden of colorectal cancer-associated genetic variants is more strongly associated with early-onset vs late-onset cancer. Gastroenterology 2020;158:1274-86.

11 Perea J, Rueda D, Canal A, et al. Age at onset should be a major criterion for subclassification of colorectal cancer. J Mol Diagn 2014;16:116-26.

12 Gupta S, Bharti B, Ahnen DJ, et al. Potential impact of family historybased screening guidelines on the detection of early-onset colorectal cancer. Cancer 2020;126:3013-20.

13 Low EE, Demb J, Liu L, et al. Risk factors for early-onset colorectal cancer. Gastroenterology 2020;159:492-501.

14 Hofseth LJ, Hebert JR, Chanda A, et al. Early-Onset colorectal cancer: initial clues and current views. Nat Rev Gastroenterol Hepatol 2020;17:352-64.

15 Holowatyj AN, Perea J, Lieu CH. Gut instinct: a call to study the biology of early-onset colorectal cancer disparities. Nat Rev Cancer 2021;21:339-40.

16 Perea García J, Arribas J, Cañete Ángel, et al. Association of polyps with early-onset colorectal cancer and throughout surveillance: novel clinical and molecular implications. Cancers 2019;11:1900.

17 Muller C, Ihionkhan E, Stoffel EM, et al. Disparities in early-onset colorectal cancer. Cells 2021;10:1018.

18 Biller LH, Ng K. Pioneering a new care model for young-onset colorectal cancer: innovations in clinical care and scientific discovery. Colorectal Cancer 2020;9:CRC18. 